\title{
Genes Encoding the Phycobilisome Rod Substructure Are Clustered on the Anabaena Chromosome: Characterization of the Phycoerythrocyanin Operon
}

\author{
RONALD V. SWANSON, $\dagger$ ROBERT DE LORIMIER, AND ALEXANDER N. GLAZER* \\ Division of Biochemistry and Molecular Biology, Department of Molecular and Cell Biology, \\ 229 Stanley Hall, University of California, Berkeley, California 94720
}

Received 15 October 1991/Accepted 10 February 1992

\begin{abstract}
The phycoerythrocyanin (pec) operon, cloned from Anabaena sp. strain PCC 7120, encodes four genes, pecBACE, located upstream of the $C$-phycocyanin (cpc) operon. This pec-cpc cluster includes all the genes for the structural components of the phycobilisome rod. Oligonucleotide probes based on the amino-terminal sequence of the phycoerythrocyanin $\beta$ subunit were used to clone an 8.0-kbp EcoRI fragment which was determined, by sequencing, to partially overlap the previously cloned $c$ pc operon. A 5.0-kbp EcoRI-ClaI fragment corresponding to the region upstream of the $c p c$ operon was subsequently subcloned and sequenced. Five open reading frames whose polarity of transcription is parallel to that of the $c p c$ genes were identified. pecB and pec $A$ encode the $\beta$ and $\alpha$ subunits of phycoerythrocyanin, respectively. pec $C$ encodes the phycoerythrocyanin-associated linker polypeptide $L_{R}{ }^{34.5, P E C}$. The identities of these genes are confirmed by agreement with amino-terminal sequences determined from purified phycobilisome components. A gene homologous to $c p c E$, found downstream of pecC, has been designated pecE. The $c p c E$ gene product is involved in the attachment of the phycocyanobilin chromophore to the $\alpha$ subunit of phycocyanin. Three transcripts were observed by Northern (RNA) analyses. The most abundant of these transcripts, $1.35 \mathrm{kbp}$, corresponds to the $\beta$ and $\alpha$

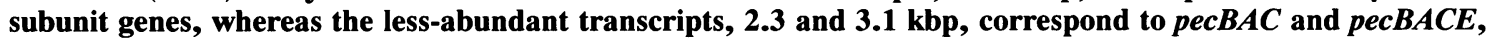
respectively. Phycoerythrocyanin is strongly induced in cells cultured under low light. In parallel, all three transcripts were present at much higher levels in cells cultured under low light.
\end{abstract}

Phycobilisomes are very large protein complexes which function as light-harvesting antennae in cyanobacteria and red algae. The proteins which compose these highly ordered structures fall into two groups: phycobiliproteins and linker polypeptides. The phycobiliproteins are a large family of colored proteins which serve as the major light-harvesting components of the phycobilisome. These proteins absorb visible light by virtue of bilin prosthetic groups, linear tetrapyrroles covalently attached to the protein through thioether linkages to cysteinyl residues. The phycobiliproteins are subdivided into three major groups, allophycocyanins, phycocyanins, and phycoerythrins, on the basis of sequence homology, bilin content, and location in the antenna complex. The linker polypeptides, which also constitute a homologous family, mediate the ordered interactions of the phycobiliproteins to form the phycobilisome. The phycobilisome is composed of two distinct subdomains: the core, which contains primarily allophycocyanin, and the peripheral rods, which contain phycocyanin and, when present, phycoerythrin. Energy absorbed by the rods, which contribute the majority of the absorption cross section of the phycobilisome, is efficiently transferred to the core from which it migrates to the reaction centers in the thylakoid membrane.

Allophycocyanin invariably contains only phycocyanobilin (PCB). The absorption spectrum of allophycocyanin varies little from one organism to another. In contrast, depending on organismal source, the rod phycobiliproteins, the phycocyanins and phycoerythrins, vary widely with

\footnotetext{
* Corresponding author.

$\dagger$ Present address: Department of Biology, California Institute of Technology, Pasadena, CA 91125.
}

respect to the types of bilins they carry and, consequently, with respect to spectroscopic properties. The four bilins present in various combinations in cyanobacterial and red algal phycocyanins and phycoerythrins have distinctive absorption spectra. Protein-bound PCB, phycobiliviolin, phycoerythrobilin, and phycourobilin absorb maximally at 620 , 568,565 , and $490 \mathrm{~nm}$, respectively.

Phycocyanins are composed of two polypeptide chains: the $\alpha$ subunits $(\sim 162$ residues) carry one bilin chromophore at position $\alpha-84$, and the $\beta$ subunits $(\sim 172$ residues) carry two bilins, one each at positions $\beta-82$ and $\beta-153$. Most species of freshwater cyanobacteria produce $\mathrm{C}$-phycocyanin (CPC), which carries PCB at all three positions. Many red algae produce R-phycocyanin, which carries the shorterwavelength-absorbing phycoerythrobilin at the $\beta-153$ position and PCB at the other two positions (7); several marine Synechococcus spp. have been found to synthesize R-phycocyanin II, which carries phycoerythrobilin at both the $\beta-153$ and $\alpha-84$ positions and PCB at $\beta-82$ (23). At least one marine Synechocystis strain synthesizes a phycocyanin which carries phycourobilin at position $\alpha-84$ and PCB at $\beta-82$ and $\beta-153$ (27).

Several cyanobacterial strains which contain multiple sets of $c p c$ genes are known. In some of these organisms, the additional phycocyanin is induced by changes in light quality, as part of a chromatic adaptation response. Calothrix sp. strain PCC 7601 contains three distinct CPCs: one is constitutive, another is induced in red light, and the third, which lacks nonessential cysteine and methionine residues, is induced under conditions of sulfur limitation $(21,22)$. A relatively small number of cyanobacteria, made up primarily of filamentous heterocyst-forming strains, synthesize both $\mathrm{CPC}$ and phycoerythrocyanin (PEC). The latter protein 
carries a phycobiliviolin at position $\alpha-84$ and two PCBs on the $\beta$ subunit $(6,7)$. PEC is induced under low-light conditions and occupies the core-distal position in the rods of phycobilisomes. The strains which produce both CPC and PEC are unique in that they synthesize two phycocyanins with different bilins within the same cell and consequently present a special opportunity for studying questions of discrimination between bilins in the course of bilin attachment during phycobiliprotein biosynthesis.

PEC was discovered and originally characterized in two strains of Anabaena (6). Subsequently, the protein from Mastigocladus laminosus PCC 7603 has been thoroughly characterized. The amino acid sequence of $M$. laminosus PEC has been completely determined (15), and its threedimensional structure has been solved by X-ray crystallography (13). Recently, a partial sequence of the pec operon from this organism has been reported (14). The pec operon sequence of Anabaena sp. strain PCC 7120 described here adds to the body of data on PEC and to that on phycobiliprotein genetic organization in general. This clone also provides a powerful substrate for genetic manipulations aimed at dissecting the mechanisms of bilin addition and discrimination between bilins, as well as for exploring the molecular basis of regulation by light intensity.

\section{MATERIALS AND METHODS}

Strains and culture conditions. Anabaena sp. strain PCC 7120 was cultured in BG-11 medium (1) under constant illumination, with either high $\left(200\right.$ microeinsteins $\left.\mathrm{m}^{-2} \mathrm{~s}^{-1}\right)$ or low (40 microeinsteins $\mathrm{m}^{-2} \mathrm{~s}^{-1}$ ) light, at room temperature or at $36^{\circ} \mathrm{C}$, as indicated in the text. Cultures were aerated by bubbling with $4 \% \mathrm{CO}_{2}-96 \% \mathrm{~N}_{2}$ or with $4 \% \mathrm{CO}_{2}-96 \%$ air. Escherichia coli DG-98 (thi-1 endA1 hsdR17 supE44 lacI ${ }^{\mathrm{q}}$ lacZ $\Delta M 15$ proC1::Tn10 $\mathrm{F}^{\prime}$ lacI $^{\mathrm{q}}$ lacZ $\Delta M 15$ proC ${ }^{+}$), obtained from Cetus Corp., was used for cloning of recombinant plasmids. DG-98 was cultured in Luria-Bertain: (LB) liquid or on LB agar plates, supplemented with $100 \mu \mathrm{g}$ of ampicillin $\mathrm{ml}^{-1}$ when appropriate.

Isolation of PEC subunits for sequencing. PEC was purified from low-light room-temperature cultures by the procedure of Bryant et al. (6) for the purification of PEC from Anabaena sp. strain PCC 6411 . Subunits were separated by reverse-phase chromatography on a C-4 column (26). After chromatography, solvent was evaporated under a stream of $\mathrm{N}_{2}$, and the subunits were redissolved in $44 \%$ formic acid. Amino-terminal sequencing was performed with an automated gas-liquid phase sequenator (Applied Biosystems, Inc.) by using $400 \mathrm{pmol}$ of the $\beta$ subunit.

The $\alpha$ subunit sequence was obtained from material purified by an abbreviated method. Anabaena sp. strain PCC 7120 cells $(70 \mathrm{mg})$ were resuspended in $0.7 \mathrm{ml}$ of $20 \mathrm{mM}$ 2-mercaptoethanol by pipetting, $0.7 \mathrm{ml}$ of $9 \mathrm{M}$ urea (pH 2.0) was added, and the solution was mixed by gentle inversion and incubated at room temperature for $30 \mathrm{~min}$. The solution was spun in a microfuge for $2 \mathrm{~min}$, and the supernatant was removed to a clean tube and spun again for $10 \mathrm{~min}$. The supernatant $(300 \mu \mathrm{l})$ was injected directly onto the C-4 column and chromatographed according to the subunit separation procedure. Then, $650 \mathrm{pmol}$ of $\alpha$ subunit was recovered and sequenced.

Isolation of chromosomal DNA. Chromosomal DNA was prepared from Anabaena sp. strain PCC 7120 cells harvested by centrifugation and resuspended in $50 \mathrm{mM}$ Tris $(\mathrm{pH}$ 8.0)-100 mM EDTA-10\% (wt/vol) sucrose. This suspension was frozen and thawed three times, lysozyme was added to
$10 \mathrm{mg} \mathrm{ml}^{-1}$, and the suspension was incubated at $37^{\circ} \mathrm{C}$ for 30 min. At this point, pronase and sodium dodecyl sulfate (SDS) were added to final concentrations of $1 \mathrm{mg} \mathrm{ml}^{-1}$ and $1 \%$ (wt/vol), respectively, and the incubation at $37^{\circ} \mathrm{C}$ was continued for $60 \mathrm{~min}$. The lysate was extracted sequentially with phenol and chloroform. Finally, DNA was purified by buoyant density centrifugation in a cesium chloride gradient.

Cloning and sequencing. Two 128 -fold-degenerate 17 -nucleotide (nt) oligonucleotides were synthesized on the basis of two overlapping segments of the amino-terminal sequence of the PEC $\beta$ subunit, probe $B 2$ with the sequence $5^{\prime}$ G-C-T/ C-T-G-T/C-T-C-N-A-C-N-A-C-T/C-T-T3' and probe B4 with the sequence $5^{\prime}$ G-A-T/C-G-C-N-T-T-T/C-T-C-N-A-A-A/GG-T3'. These oligonucleotides were end labelled with ${ }^{32} \mathrm{P}$ by using T4 polynucleotide kinase and used to probe Southern blots of HindIII digests of chromosomal DNA. Hybridizations were performed according to the conditions described by Wallace and Miyada (29) at $46^{\circ} \mathrm{C}$ for probe $\mathrm{B} 2$ and at $42^{\circ} \mathrm{C}$ for probe $\mathrm{B} 4$. Washes were done at room temperature in 0.9 $\mathrm{M} \mathrm{NaCl}-0.18 \mathrm{M}$ Tris (pH 8.0)-6 mM EDTA-0.5\% SDS. Both probes hybridized to a 1.3-kbp fragment. A library was constructed from chromosomal DNA, which had been cut with HindIII and sized on a $1 \%$ low-melting-point agarose gel, and the vector pBluescript KS + (Stratagene), which had been cut with HindIII and treated with calf intestinal alkaline phosphatase. Transformants of strain DG-98 were pooled into 20 groups of $\sim 30$ each by being resuspended in LB medium. Plasmid DNA was isolated from these pools by the alkaline lysis method. A portion of each of these plasmid pools was probed by using the dot blot technique described by Costanzi and Gillespie (10). One positive pool was identified, and a small aliquot of the remaining DNA was used to transform $E$. coli DG-98. The dot blot procedure was repeated on 60 individual colonies from this transformation, and two positive clones were recovered. Restriction mapping of these clones suggested that they carried the same insert. Partial sequencing of one clone, $\mathrm{pF} 10$, revealed that it carried a portion of pecB. A 19-nt oligonucleotide, probe B5 with the sequence 5'T-C-A-T-C-T-C-C-A-C-T-A-A-G-A-TA-A-T3', was synthesized, on the basis of the sequence of $p e c B$, for use as a sequencing primer and probe. On the basis of restriction mapping of pF10, an EcoRI digest of chromosomal DNA was blotted and probed with $\mathrm{B} 5$ at $48^{\circ} \mathrm{C}$. The single $8.0-\mathrm{kbp}$ fragment identified by this procedure was cloned in pBluescript by a method similar to that described above to give clone pF400. Double-strand sequencing of pF400 HindIII, HincII, and Sau3A subclones was done by the dideoxy method (24) by using the Applied Biosystems dye primer cycle sequencing kit and Model 373A automated DNA sequencer. Some sequences were obtained with the Sequenase Version 2.0 sequencing kit (U.S. Biochemicals) by using pF500 primed from oligonucleotides complementary to the insert.

RNA isolation and Northern (RNA) blotting. RNA was isolated according to Golden et al. (18) from cells grown under either high (200 microeinsteins $\left.\mathrm{m}^{-2} \mathrm{~s}^{-1}\right)$ or low (40 microeinsteins $\mathrm{m}^{-2} \mathrm{~s}^{-1}$ ) light at $36^{\circ} \mathrm{C}$. No significant contamination of the purified RNA by DNA was detected. Cultures were diluted twofold with fresh medium every 12 to $24 \mathrm{~h}$ to maintain an optical density at $550 \mathrm{~nm}$ of 0.2 to 0.6 . This resulted in a maximum variation of 1.5 -fold in the amount of light transmitted by a culture. Before being harvested, cultures were grown continuously for at least four generations under these conditions. Culture doubling times were 11 $\mathrm{h}$ under high light and $24 \mathrm{~h}$ under low light.

For Northern blotting, absolute amounts of high- and 


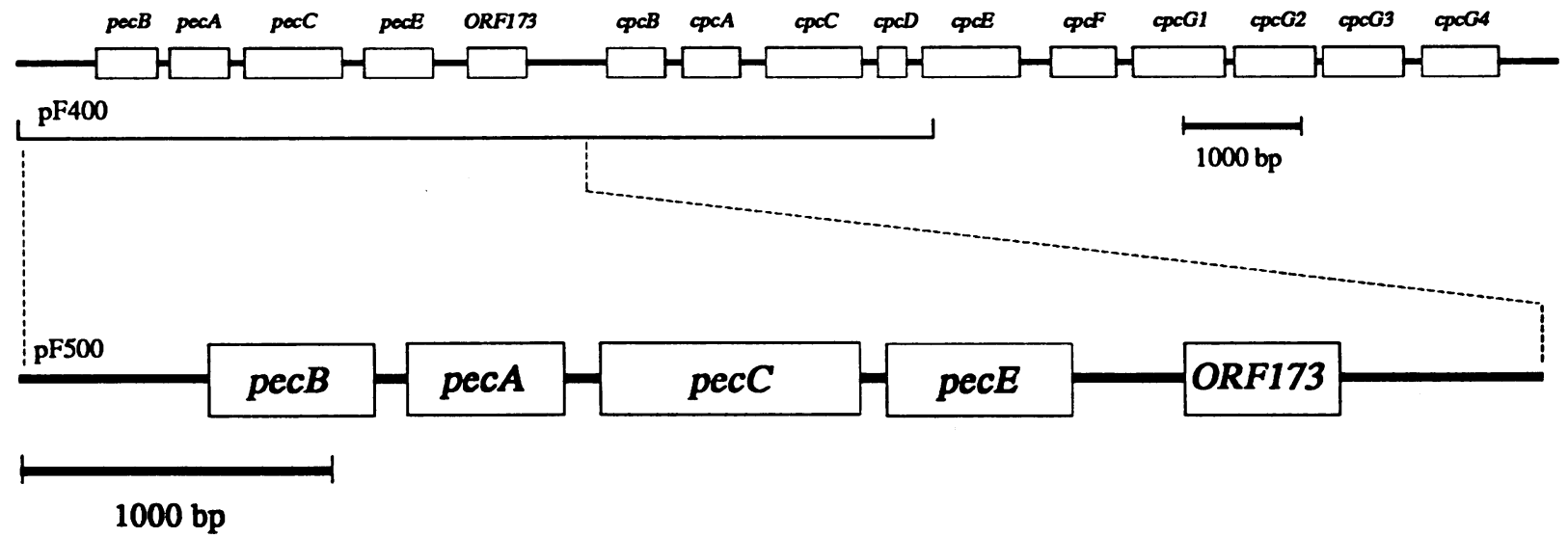

FIG. 1. Genetic organization of the pec and $c p c$ loci on the chromosome of Anabaena sp. strain PCC 7120. The upper portion of the diagram represents the linkage and orientation of the two operons relative to one another; the extent of clone pF400 is shown directly below this. The lower portion shows an enlarged region corresponding to the EcoRI-ClaI subclone pF500. The diagram was compiled from data presented in this paper as well as data from references 3 and 8 .

low-light RNA were matched to within $5 \%$ by $A_{260}$. RNA was fractionated by electrophoresis in a $1.2 \%$ agarose gel containing $6 \%$ formaldehyde-50 mM HEPES $(N-2$-hydroxyethylpiperazine- $N^{\prime}$-2-ethanesulfonic acid [pH 7.2])-1 $\mathrm{mM}$ EDTA and transferred to nitrocellulose. Filters were probed with restriction fragments which had been ${ }^{32} \mathrm{P}$ labelled by using the random primed DNA labelling kit from Boehringer Mannheim. The gene-specific probes used were as follows (numbering according to Fig. 2): pecBA, HindIII fragment (nt 1102 to 1842); pecC, Sau3A fragment (nt 2318 to 2677); pecE, XmnI-HindIII fragment (nt 2900 to 3267); ORF173, $A c c$ I fragment (nt 4090 to 4460 ); and $c p c B A, X b a \mathrm{I}-B s s \mathrm{HII}$ fragment corresponding to nt 315 to 712 of the $c p c$ operon (numbering according to reference 3 ). Relative levels of RNA species were quantitated by two methods. In the first, individual bands were excised from the nitrocellulose filters of Northern blots and quantitated by scintillation counting. The second method utilized dot blots of serially diluted RNA samples from high- and low-light cultures. Identical sections of the filter were probed with either the $p e c B A$ or $c p c B A$ restriction fragments. Dot blots were autoradiographed without intensifying screens, and the resulting films were scanned with a densitometer. Peak areas from triplicate samples were used to quantitate relative RNA levels.

Nucleotide sequence accession number. The nucleotide sequence reported in this paper has been submitted to GenBank and assigned the accession number M80357.

\section{RESULTS AND DISCUSSION}

Isolation of the PEC genes. PEC was purified from lowlight cultures of Anabaena sp. strain PCC 7120 grown at room temperature, as previously described (6). The $\alpha$ and $\beta$ subunits were separated by reverse-phase high-performance liquid chromatography (HPLC) (26), and amino-terminal sequences were determined to provide information for the design of oligonucleotide probes for cloning. Two degenerate probes based on the $\beta$ subunit sequence were synthesized and used to probe Southern blots of HindIII-digested chromosomal DNA. While each probe hybridized to several bands with approximately equal affinity, only one band was recognized by both probes. This $1.3-\mathrm{kbp}$ fragment was cloned and found by sequencing to contain the $5^{\prime}$ end of the $p e c B$ gene and a single EcoRI site $750 \mathrm{bp}$ upstream of the start codon of pecB. On the basis of these observations, a nondegenerate oligonucleotide was synthesized and used to probe an EcoRI digest of chromosomal DNA. The sole hybridizing fragment $(8 \mathrm{kbp})$ was cloned. This clone, pF400, was determined, by partial sequencing, to overlap the previously cloned $c p c$ operon (3) as shown in Fig. 1. An EcoRI site used for cloning this fragment was shown to lie within the $c p c E$ gene by sequencing. The fact that it is not present in the $c p c$ operon sequence reported previously suggests a polymorphism between laboratory strains. The replacement of a $\mathrm{T}$ by a $\mathrm{C}$ at position 2515 in the $c p c$ operon (using the numbering from reference 3 ) does not result in a change in the translated amino acid sequence.

These results, along with recent further characterization of the $c p c$ operon which showed that it contains a small gene family encoding the rod-core linkers (8), show that all the known structural components of the phycobilisome rod are encoded within the 13-kbp region of the Anabaena chromosome depicted in Fig. 1. The close linkage of the pec and cpc operons is reminiscent of the clustering of some phycobiliprotein genes in other cyanobacterial species such as Calothrix sp. strain PCC 7601, which contains a cluster encoding allophycocyanin, inducible-phycocyanin, and constitutivephycocyanin operons (9), Synechococcus sp. strain PCC 6301, which carries two closely linked phycocyanin operons (19), and marine Synechococcus spp., which have recently been found to possess a cluster containing two phycoerythrin operons along with an R-phycocyanin II operon (12). It is unclear whether these clusters represent a residual consequence of the evolution of these genes by duplication and subsequent divergence or whether they may have some functional significance. It should be noted that many examples of phycobilisome genes which are not clustered are known and that genomic mapping indicates that at least two of the phycobilisome core components in Anabaena sp. strain PCC 7120 are not closely linked to one another (2).

Nucleotide sequence of pF500 and identification of pec genes. On the basis of the restriction map of the $c p c$ operon, a 5-kbp EcoRI-ClaI fragment representing the region $5^{\prime}$ to the $c p c$ genes was subcloned and sequenced. The nucleotide sequence of the entire EcoRI-ClaI insert of pF500 is shown in Fig. 2. The region from nt 4711 to 4996 overlaps the previously reported $c p c$ operon sequence (3), and no dis- 
1 GAATTCTGCTTTAACGTGAGTCTCCAACGGAGGCGCACGTTGCTTTAACCTCCGTGTCTCTAAAAAATGAGAATCAAAAAAAATTTTCGATGTTTTGTTACTCAAATTCACAAAACATTA 121 CATATAGGTTTTTCCTGATAAATCAAATAATTGCTAGTAATAATGCGCGAATCGCTTATAAACAGCAGTTAACAGGCTATTAATAATTATGATGAATATTAGTCTTGAAAATTATTATCA 241 TTTTTAGCCACAAAATAAAAACCGAAGATTGTTTATCTAAAACACAGATTTTTTTTGATAAAAATCCTGCTTTAGCTTTGTTTATCAATTTCTACTGTCAGCATGAAAGTATATATAAGC 361 TAGATTTGCTACCGCCGTAGAMATTATACTCAAGCATTTATTAAGTAAAGTAAACAATATTATTTAATTGTTAAACGGTTTTTCACCTAAAGGCATTACAACCTTTATAAAACAATAATT 481 TTTAGAGAAGTCTGTATGGATTAACACTATCTTACAAAACTTAATAATAAATTCACTCAAGCCATATATAAAATACGAACTAAGGTTTTCAAAGCAATTAAAAAACAGAAAACAAATTGC 601 TGTTTTCCTAATAAGAAATCGCACATGATTGCAGTCCCCCAAGATAATATATGGGAGTTAATTCTGCTTGAATATTTGTTTTCTAAATAGTAAGAATAATTGCAATCGACCTTATAAAAA PeCB> $\mathbf{Z}$ : D

841 TTGCTGATAGTAATAAGCGGTTAGATATAGTTAACAGACTAACAAGTAATGCTTCCTCCATTGTGGCTAATGCTTATCGTGCTTTAGTTGCTGAACGTCCACAAATATTTAATGCTGGTG 961 GTGCTTGTTTCCACAATCGTAACCAAGCTGCTTGTATCCGTGATTTAGGATTTATTCTGCGTTATGTCACCTATTCTGTATTAGCAGGTGATGGTAGTGTCATGGACGATCGCTGCTTAA

1081 ATGGTCTGAGGGAAACATATCAAGCTTTAGGTACTCCTGGCGATGCAGTAGCATCTGGAATCCAAAAAATGAAAGATGCAGCAATTGCAATTGCTAATGACTCCAAAGGCATTACTAAAG 1201 GTGATTGTAGTCAGTTAATTGCTGAATTAGCTAGCTATTTTGATCGCGCTGCTTCTGCGGTTGTTTAAATAGATGTTTTTTCCAAAACTTTTTTCAAACTGACTGTTAGTAATTAATCGC D C S Q I I A E I A S Y F D R A A S A V V 1321 AAATCAAGGAGATTAGGAGAGTATGAAAACACCTTTGACGGAGCGATCTCTGCTGCTGATGTTCGTGGTTCCTACCTCAGCAACACTGAAATGCAAGCAGTTTTTGGTCGTTTTAACC

1441 GCGCTCGCGCTGGTTTAGCGGCTGCTCAAGCATTTTCTAATAATGGCAAAAATGGGGAGAGGCAGCAGCAAATCATGTTTATCAAAAATTCCCTTACACCACTCAAATGAGTGGGCCTC 1561 AATATGCTTCCACTCCAGAAGGTAAATCCAAGTGTGTTCGTGATATTGACCACTATCTTCGTACTATTAGCTATTGCTGCGTAGTCGGTGGTACTGGCCCCTTAGATGAATATGTAGTAT Y A S T P E G K S K C V R D I D F Y I R T I S Y C C V V G G T G

1681 CTGGTTTAAGCGAACTCAACAGCGCCCTTGGTTTATCTCCTAGCTGGTATGTAGCTGCACTAGAATTTGTCCGGGATAATCACGGTTTAAATGGTGATGTTGCAGGTGAAGCTAATATCT $G$ I $S$ E 1801 ACCTGAACTATGCAATTAACGCTTTAAGTTAAGACAAAGCTTGTGGGTCTTGGGAAATAGGTAATTGCTCAATTTAAGTGAGTTACTTTGTAACTGCTTTGAACATAATTGCAGCATTTT

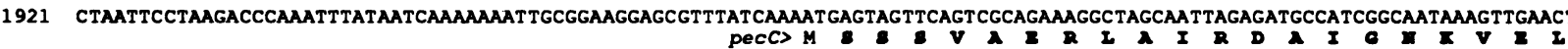

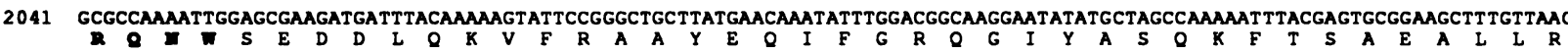
2161 AAATGGCAAAATCAGTGTGCGGCAATTTGTAGAGATTTTAGCCAATCTGAATT TTACAAAGAATGCTTTTTTTAATAAAAACTCTCAAGTGCGTTTGATTGAATTAAACTATAAGCACTT 2281 ACTGGGACGCGCACCGTACGATCAATCAGAGATTGCTGATCATGTAGACATTTATGCTGCTCGTGGCTACGATGCTGACATTGATGCTTACATTTACAGTTCAGAaTACGAAATGCTTT

2401 TGGTAATTCTATTGTTCCTTATTACCGAGGATTCCAATCAATCCCTGGAATGAAAACAGTAGGATTTAATCGTATATGTGAGCTTTATCGTGGTCGGGGTAATAGCGATAACGCACAAAT GGGACGGACAAATTCTCGTTTACGAACAAAAGTTTCCCTGAATTTGCCTAATGGAATTTTGCCACCAACTTCCGCCGGGACAAACTTTGTTTCCGCAGCACCGACGTTAATCAGTTCTGC

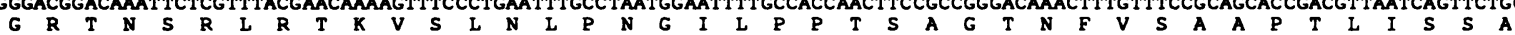
2641 GACGAAGGGTGATAATCGGATGTTTGTGATTGAAGCGATCGCCGGTGGATTGAATACAAACGTAGCAGTCCGTCGCAGCCGCCAGGTTTACACCGTTTCCTATGAAAGACTCTCCGCCAC

2761 TTACCAAGAAATTCATAAGCGTGGTGGAAAGATAGTCAAGATTTCCCAAGTGTAGAAATGGTTGCTAGATACCCAACTTCTTGAAGAAGTGGGGTATCTGAGTAAATTTTGAATTTCGGA 2880 $Y$ O E I H K R G K I V K I S Q V

2881 GCAAAGCAACGTGACTGCTGAACCAATTCTTTCTCCAGAAACAGCGATCGCAGCTTTATCTGGTGAGGATAACCAAATTCGTTATTATGCTGCTTGGTGGTTGGGTAAACATAACGTACA 3000 pecE> V T A E P I L S P E T A I A A L S G E D N Q I R Y Y A A W W L G K H N V Q

3001 GGCTGGTTTTACGGCGCTATGTGTAGCCTTATTTGATGAACGCTATCGCATCCCATCAGGAGGGTATCCTCTGCGTCGTCAGGCTGCACGGGCTTTAGGATTGTTGAAAAATCCCCAAGC 3120

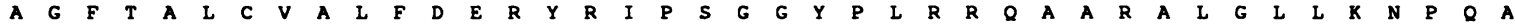

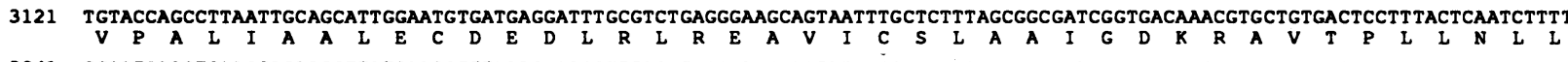

3241 GCAATCAAGTCAAGCACAACCTTATGAAGCTTTAATCGAAGCTTTAGCTACTCTACAAGTTTGGTCAGCCCGTCCACAAATTGAACCTTTTCTTCAGCATTATTCTGAGCGTGTGCAAT

3361 TGCAGCCGCTAGATATATGTATCTCCTCACCCAAGATTCTGAATACATTGAaCGTATTGTCAAAAATCTCAATCATGACATATGTATT TACGTTGGGCGGCGGTATTTGATTTGGGTGC

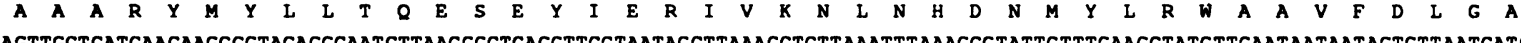
3481 AGTTGCTCATCAACAAGCCGTACAGGCAATCTTAACGGCTCAGGTTCCTAATAGCTTAAAGCTCTTAAATTTAAAGCGTATTCTTTGAAGCTATGTTGAATAATAATAGTGTTAATCATG 3600 $\begin{array}{llllllllllllllllllllllllllll}V & A & H & Q & Q & A & V & Q & A & I & L & T & A & O & V & P & N & S & L & K & L & L & N & L & K & R & I & L\end{array}$

3601 AAAAAGCAGCTTTATTATTTGGAGCAATTGATGATTTATTAATTCAACTTTAAAACTCATAACTGAATATTGGGGATTGGGGATTGGGGATTGGGGATTGGGGATTGGGGATTGGGGATT 3720

3721 GGGGATTGGGGATTGGGGATTGGGGATTGGGGATTGGGGATTGGGGATTGGGGATTGGGGATTGGGGATTGGGGATTGGGGATTGGGGATTGGGGATTGGGGATTGGGGATTGGGGATTG 3840

3841 GGGATTGGGGATTGGTTTGTTATTAATATCTCCAGTCCTTTTTGTGTTGAATTTAGGACT TACGCACTGAACCAAAAAAAACTTCTGAAGATGGTCAATAGTCAATAGTCCACAGTCCAA 3960

3961 AAAACTTGATTTTAACTATTGACTATAAATGTTTGAAACCCCTACACCCGGTCTCAACAGAAAATCTGGGTGCGTAATTCGTAGCATTGTTTTATCCAGAAATAAATTATGAATCAAGCT 4080 ORF173> M N Q A

4081 TCATTGAGCGTAGACGCTATTACCAATTTAATCGAAGCATTTCATCATCACCATCCTGCTGTTAGAAGTGCGGCTGTAGATGAATTAATCAAATTGGGAGCATCACAGTTAATTTGTTA 4200

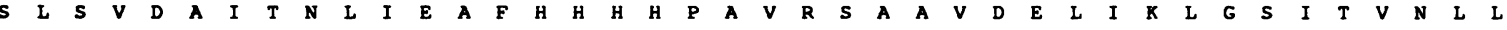

4201 ATTGCTGCTTATGATGATTCTCAAGATCAAGGTTTTCAAGCCCAGATCATTCAGGTTTTAGCTCAAATTGGTGATGCAAAGGCACTAAAATTATTAGCCGAGGTGGTGGGGACAAGTGTA 4320

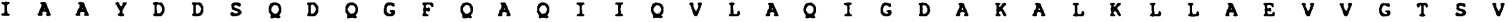

4321 GCCAATCACTGTCAAGGTAATGTCCGCCGCATTGCTGCACGAGGGTTAGGAAAAATCGCCAGCACCACTAGTAATACAGAAATAATCAACAATGCTCAAGAAAAGTTAATTTGGGCATTA 4440 $\begin{array}{lllllllllllllllllllllllllllllllllllllllll}A & N & H & C & Q & G & N & V & R & R & I & A & A & R & G & L & G & K & I & A & S & T & T & S & N & T & E & I & I & N & N & A & Q & E & K & L & I & W & A & L\end{array}$

4441 CTTACCCCCGAAGATTGGGGTCTACGTTACGCTGCTGCTGTCTCTTTACAAGAAATTGCCACGCCAAAAGCTAAAGCTGCTTTACAACAAGCGATCGCCCAAGAAACCGATCCAGTTGTT 4560

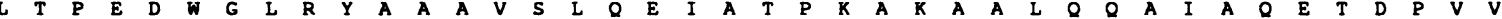

4561 CGCTCACGTATGGCGATCGCCTTGAGTTAGGGAACAGAGAGCAAGGGGGAGAAACTTAAAGATTTCCCCAATCCCAATTCCTCATCTCTCATCCCTGGAAATGTTGCGAAAGATTAATTT 4680 R S R M A I A L S

4681 TACGTTTATTCACAAAAACCATCCTGTGGAAAAAAATGGCGTAAAACAGCAATAGATTGTCACCACTAGGTTTTTGCAACTATTAAAAAATAATTAAGACACCAATATTTTGCCTAAAAT 4800 4801 CACTAATTCATGAAGTTATGTTACGGGAGAAGGGGTTTTTCTCAGTTCTAGCCCTTGTATGTAAAGCATTTGAGGGGGTTCTTATTCATGCAACTTATTTATTCACAATTTGTAACAAA 4920

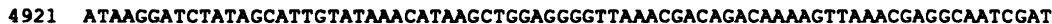

FIG. 2. Nucleotide sequence of the 4,996-bp EcoRI-ClaI insert of pF500. The translated amino acid sequences of the pec genes as well as that of ORF173 are presented.pecB, encoding the PEC $\beta$ subunit, extends from nt 750 to 1265. pec $A$, encoding the PEC $\alpha$ subunit, extends from nt 1344 to 1829 . pecC, encoding the PEC-associated linker polypeptide $\mathrm{L}_{\mathrm{R}}{ }^{34.5, \mathrm{PEC}}$, extends from nt 1979 to 2812 . pecE, encoding a polypeptide with homology to $c p c E$, extends from nt 2891 to 3565 . Residues of these three gene products which have been confirmed by $\mathrm{N}$-terminal sequencing are indicated by bold type. Amino acid sequence data for $\mathrm{L}_{\mathrm{R}}{ }^{34.5, \mathrm{PEC}}$ is from reference 8 . ORF173 extends from nt 4069 to 4587. The sequence was determined on both strands, except for the two end regions from nt 1 to 78 and from nt 4929 to 4996 , which were determined on a single strand. DNA sequencing data were managed and analyzed with the IntelliGenetics suite of programs. 
PEC ALPHA

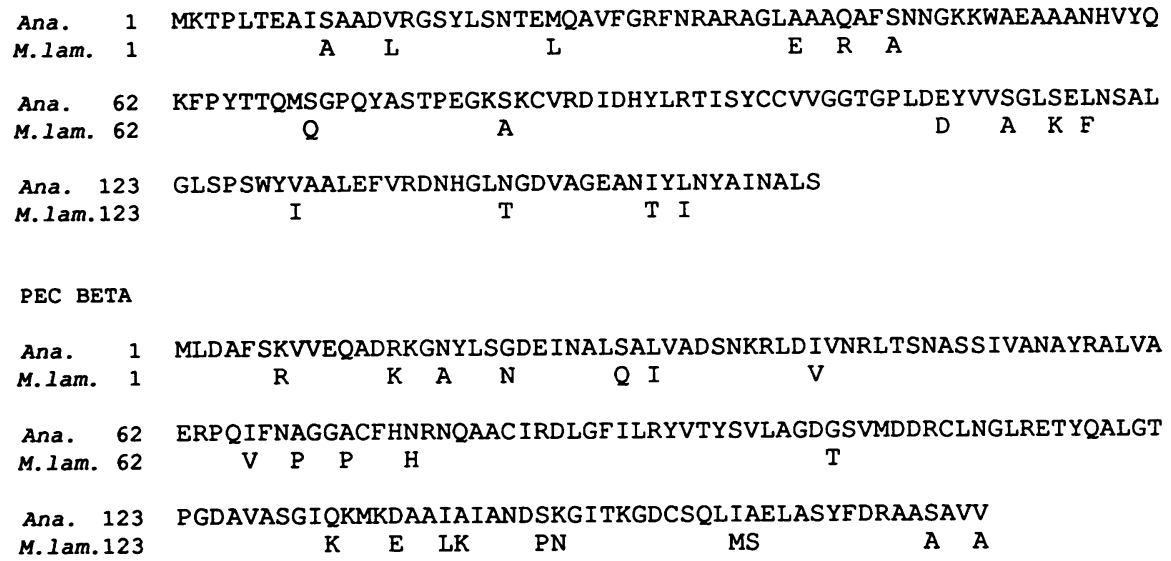

FIG. 3. Deduced amino acid sequences of the $\alpha$ and $\beta$ subunits of Anabaena sp. strain PCC 7120 (Ana.) PEC. Amino acid substitutions in the PEC from M. laminosus PCC 7603 (M. lam.) are shown below the sequences $(14,15)$. Blank positions indicate identity.

crepancies between the two data sets were noted in this region. Five long open reading frames ( $\geq 100$ amino acids) whose polarity is parallel to that of the $c p c$ genes are present. $p e c B$ and pec $A$ are separated by a 78-bp intergenic region and can be identified as the structural genes for the $\beta$ and $\alpha$ subunits of PEC, respectively, by agreement of the translated sequences with the amino-terminal sequences determined for these polypeptides (Fig. 2), as well as by the very high homology to the PEC subunits from M. laminosus, as shown in Fig. 3. The level of homology between the PECs of these strains, amino acid identity of $90 \%$ for the $\alpha$ subunits and $87 \%$ for the $\beta$ subunits, is similar to that seen between the CPCs from these strains, $86 \%$ for the $\alpha$ subunits and $92 \%$ for the $\beta$ subunits. A 149-bp intergenic region follows the pec $A$ gene and encodes a potential RNA stem-loop structure with a calculated free energy of formation of $-19.4 \mathrm{kcal}$ (ca. $-81.2 \mathrm{~kJ}) \mathrm{mol}^{-1}$.

This intergenic region is followed by pec $C$, which encodes the PEC-associated linker polypeptide $\mathrm{L}_{\mathrm{R}}{ }^{34.5, \mathrm{PEC}}$. This assignment is confirmed by agreement with the amino-terminal sequence determined by Bryant et al. (8) (Fig. 2). The arrangement of genes for phycobiliprotein subunits and linkers is also seen in phycocyanin operons from this and several other organisms. Only a partial sequence has been determined for the $\mathrm{L}_{\mathrm{R}}{ }^{34.5, \mathrm{PEC}}$ from $M$. laminosus; sequences of 57 residues at the amino terminus and the 114 carboxylterminal residues are available from protein (16) and DNA sequencing (14). When these sequences are compared with the Anabaena $\mathrm{L}_{\mathrm{R}}{ }^{34.5, \mathrm{PEC}}$, they exhibit $71 \%$ sequence identity (Fig. 4), significantly lower than the homologies between the PEC subunits from the two organisms. This trend is also seen with phycocyanin subunits and phycocyanin-associated linkers. These observations suggest that there are more constraints on the evolution of the phycobiliprotein subunits than on that of the linkers. pecC is followed by a 77-bp intergenic region which also encodes a potential RNA stemloop structure with a calculated free energy of formation of $-32.7 \mathrm{kcal}$ (ca. $-136.8 \mathrm{~kJ}$ ) $\mathrm{mol}^{-1}$.

An open reading frame encoding a polypeptide with significant homology to the Anabaena $c p c E$ gene (47\% identity, Fig. 5) lies downstream of pecC. Accordingly, this open reading frame has been named pecE. This gene is predicted to start with a GTG codon located 79 nucleotides downstream of the last codon of pecC. $c p c E$ genes have been found in the phycocyanin operons from several cyanobacterial species. Interposon mutagenesis of this gene in Synechococcus sp. strain PCC 7002 has been performed, and the resulting mutants have been found to produce phycocyanin which lacks the PCB chromophore at the $\alpha-84$ position (5). This finding suggests a role for this gene product in sitespecific bilin attachment to the $\alpha$ subunit (25). Mutants of Synechococcus sp. strain PCC 7002 (5) and of Calothrix sp. strain PCC 7601 (28) in which the $c p c F$ gene has been insertionally inactivated exhibit the same phenotype. Signif-

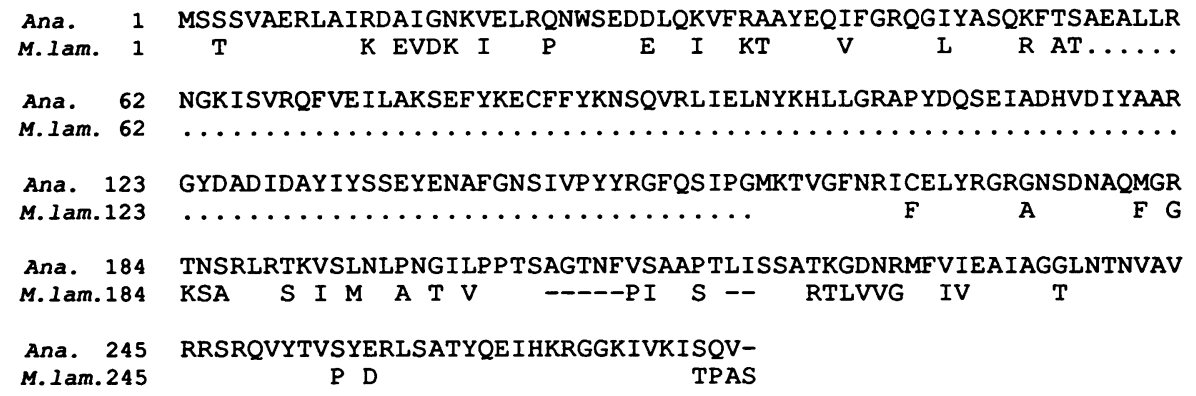

FIG. 4. Comparison of the translated amino acid sequence of pecC from Anabaena sp. strain PCC 7120 (Ana.) with the partial sequence of the M. laminosus (M. lam.) PEC-associated rod linker $\mathrm{L}_{\mathrm{R}}{ }^{34.5, \mathrm{PEC}}(14,16)$. Blank positions indicate identity with the Anabaena sequence, dashes indicate gaps introduced to increase homology, and dots indicate the undetermined region of the M. laminosus sequence. 


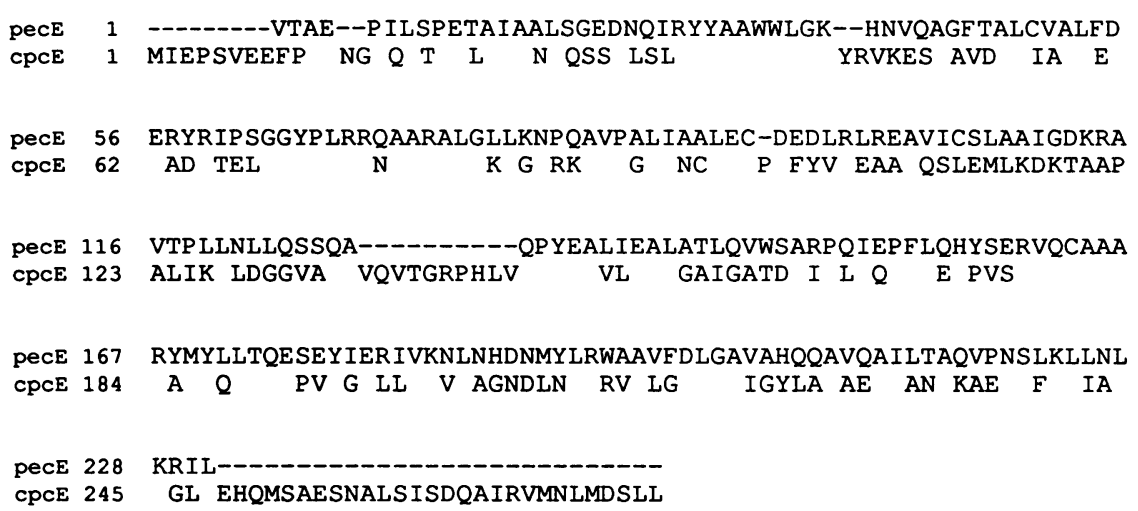

FIG. 5. Translated amino acid sequences of $p e c E$ and $c p c E$ from Anabaena sp. strain PCC 7120. The translated sequence of $p e c E$ is $47 \%$ identical to that of $c p c E$ (3). Blank positions indicate identity and dashes indicate gaps introduced to increase homology.

icantly, PEC differs from CPC in carrying a phycobiliviolin chromophore as opposed to a PCB chromophore at the $\alpha-84$ position. Consequently, pecE might play a role in discrimination between bilins or in bilin isomerization along with bilin attachment. The pecE coding region is also followed by a potential stem-loop structure.

The pec operon is followed, $104 \mathrm{bp}$ downstream of the pecE gene, by 26 direct repeats of the heptanucleotide sequence ATTGGGG. Tandem repeats of this sequence, as well as of other heptanucleotide sequences, have been observed near the coding regions of several genes cloned from heterocystous cyanobacteria (20). The significance of these repeat sequences is not known.

Following these repeats, a fifth parallel open reading frame is present, beginning at nt 4069 (Fig. 2). The 173-amino-acid sequence predicted from this open reading frame shows possible homology to $c p c F(12)$.

Identification of transcripts from the pec and $c p c$ operons. RNA was prepared from cells cultured under both high and low light for Northern blot analysis. Five probes were selected from subclones of $\mathrm{pF} 400$ representing sequences internal to $p e c B A$, pecC, pecE, ORF173, and $c p c B A$. Three major pec transcripts were detected as shown in Fig. 6. On the basis of their patterns of hybridization to specific probes, the $1.35-\mathrm{kbp}$ transcript corresponds to $p e c B A$, the $2.3-\mathrm{kbp}$ transcript corresponds to pecBAC, and the $3.1-\mathrm{kbp}$ transcript corresponds to pecBACE. As noted in the previous section, large imperfect inverted repeats are present $3^{\prime}$ of pecA, pecC, and pecE. This family of transcripts might arise either through incomplete termination at the intergenic hairpins or through partial degradation of a single large transcript. The relative abundance of each of these transcripts is depicted graphically in Fig. 6B. In addition to the three predominant segmental transcripts of the pec operon, there is a faint hybridization signal to a $2.6-$ to $2.7-\mathrm{kbp}$ species with all three pec probes. This species apparently contains pecACE, perhaps as an independently initiated transcript or as a partially degraded pecBACE transcript. There is no evidence of pecBACE transcripts longer than $3.1 \mathrm{kbp}$ which could include ORF173.

Both the pecC and ORF173 probes hybridize weakly to a $1.5-\mathrm{kbp}$ species. The size of this species and the fact that its abundance is not dependent on light intensity (see below) suggest that this signal is due to spurious hybridization to $16 \mathrm{~S}$ rRNA. No other major transcripts were observed to hybridize with the ORF173 probe. Thus, it is unclear whether this open reading frame encodes a translated prod-
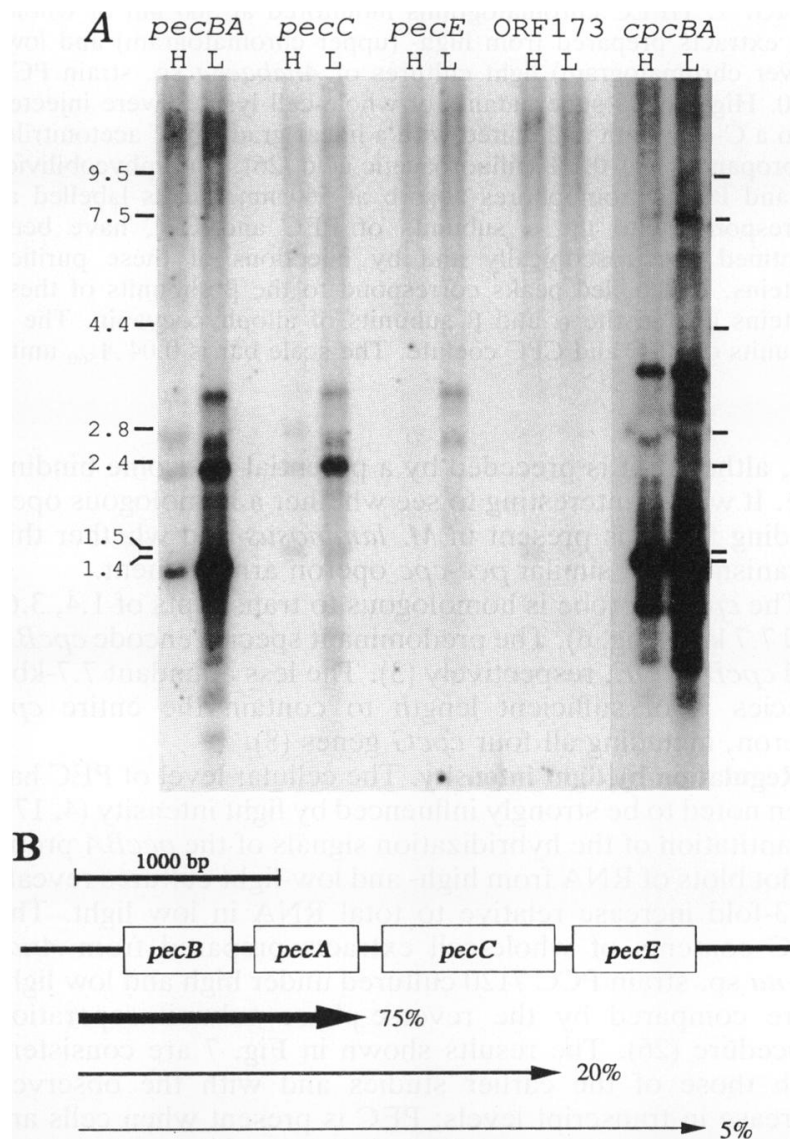

FIG. 6. (A) Transcripts of the pec and $c p c$ operons and their dependence on light intensity. A Northern blot of total Anabaena RNA from cells grown in high (H)- or low (L)-light intensity, 200 or 40 microeinsteins $\mathrm{m}^{-2} \mathrm{~s}^{-1}$, respectively, was prepared. Each pair of lanes was probed with a restriction fragment specific to the genes denoted above the blot. The positions of RNA markers (lengths in kilobase pairs) are shown to the left of the blot. The 1.5- and 2.8-kbp species were the $16 \mathrm{~S}$ and $26 \mathrm{~S}$ rRNAs, respectively, of Anabaena sp. The remaining species were from a kit supplied by Bethesda Research Laboratories. (B) pec transcripts and their relative abundance. The three major transcripts from the pec operon are depicted. Percentages refer to the relative abundance of each transcript in low light estimated from the hybridization signals on the Northern blot shown in Fig. 6 . 


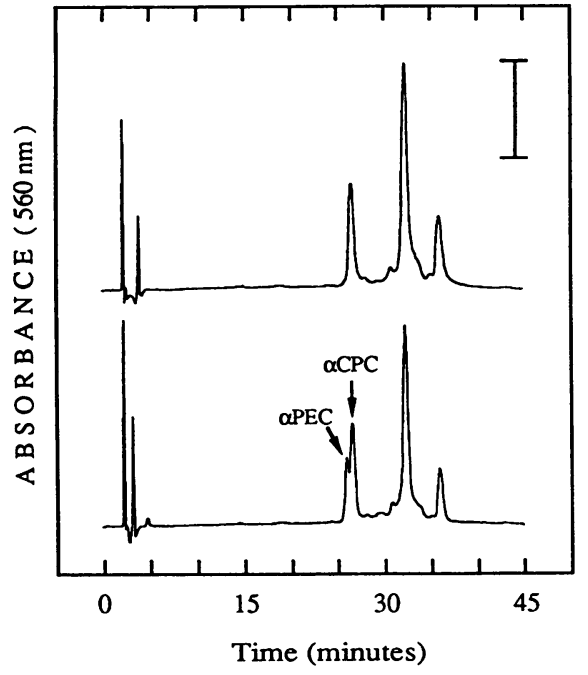

FIG. 7. HPLC chromatograms monitored at $560 \mathrm{~nm}$ of wholecell extracts prepared from high- (upper chromatogram) and low(lower chromatogram) light cultures of Anabaena sp. strain PCC 7120. High-speed supernatants of whole-cell lysates were injected onto a C-4 column and eluted with a linear gradient of acetonitrileisopropanol $(2: 1)-0.1 \%$ trifluoroacetic acid (26). Both phycobiliviolin and PCB chromophores absorb at $560 \mathrm{~nm}$. Peaks labelled as corresponding to the $\alpha$ subunits of PEC and CPC have been identified spectroscopically and by injections of these purified proteins. Unlabelled peaks correspond to the $\beta$ subunits of these proteins and to the $\alpha$ and $\beta$ subunits of allophycocyanin. The $\beta$ subunits of PEC and CPC coelute. The scale bar is $0.04 A_{560}$ unit.

uct, although it is preceded by a potential ribosome binding site. It will be interesting to see whether a homologous open reading frame is present in $M$. laminosus and whether this organism has a similar pec-cpc operon arrangement.

The $c p c B A$ probe is homologous to transcripts of $1.4,3.6$, and $7.7 \mathrm{kbp}$ (Fig. 6). The predominant species encode $c p c B A$ and $c p c B A C D E$, respectively (3). The less abundant 7.7-kbp species is of sufficient length to contain the entire $c p c$ operon, including all four $c p c G$ genes (8).

Regulation by light intensity. The cellular level of PEC has been noted to be strongly influenced by light intensity $(4,17)$. Quantitation of the hybridization signals of the pecBA probe to dot blots of RNA from high- and low-light cultures reveals a 13-fold increase relative to total RNA in low light. The PEC contents of whole-cell extracts prepared from Anabaena sp. strain PCC 7120 cultured under high and low light were compared by the reverse-phase subunit separation procedure (26). The results shown in Fig. 7 are consistent with those of the earlier studies and with the observed increase in transcript levels; PEC is present when cells are cultured under low light but is not detectable by this procedure in the high-light cultures. Because the $\alpha$ subunit of PEC is not detectable in cells grown in high light, a value for the relative increase in PEC subunits in low light was not determined. If the $\alpha$ subunit of PEC was present in high-light cells at $1 / 13$ the level in low-light cells, it would, in fact, have been undetectable in this experiment. The level of each of the three major pec transcripts increases by approximately the same factor in low light (Fig. 6), although this was not quantitated.

We observed an approximately twofold increase in $c p c B A$ transcripts in low light. This correlated with an approximate doubling of CPC, relative to chlorophyll, in cells from low-light cultures (data not shown). In contrast, Belknap and Haselkorn found that the level of $c p c$ transcripts did not change with light intensity (3). The discrepancy could be due to the relatively short time ( $12 \mathrm{~h}$ ) that these authors used to culture cells in differing light intensities and also to the use of absolute light intensities that differed from those in the present study. Belknap and Haselkorn did note that the ratio of 3.6- to $1.4-\mathrm{kbp} c p c$ transcripts varied with light intensity, increasing by a factor of 10 in low light, and they suggested that transcriptional termination between $c p c A$ and $c p c C$ was light regulated (3). We also observed an increase in the ratio of these two transcripts in low light. However, the measured increase was twofold. Whether this change is correlated with an increase in CPC-associated linker polypeptides encoded by the 3.6-kbp transcript is unknown. In the cyanobacterium Synechococcus sp. strain PCC 7002, the ratio of $c p c B A C$ and $c p c B A$ transcripts remains constant with light intensity, although the ratio of linker to CPC changes (11).

No extensive sequence similarities which might indicate regulatory regions could be detected in the $5^{\prime}$ regions of the cpc and pec operons. The interspecies comparison of the upstream regions of the pec operons from Anabaena sp. strain PCC 7120 and M. laminosus does, however, reveal similar regions located $\sim 200$ bp upstream of the translational start sites. Of the 36 nucleotides from -206 to -171 in the Anabaena sequence, 33 match the equivalent sequence from -196 to -161 in the M. laminosus sequence. Further experiments will be required to delineate the control elements of these promoters.

\section{ACKNOWLEDGMENTS}

We thank Sigurd Wilbanks of this laboratory for performing amino-terminal sequence analyses, Srini Kadambi and Michael Moore of the University of California at Berkeley Microchemical Facility for the generous gift of all oligonucleotides used in this work, Victor Boyartchuk of the Lawrence Berkeley Laboratory Human Genome Center for help with automated DNA sequencing, and D. A. Bryant for providing us with a preprint of reference 8 and sharing data with us prior to publication.

This research was supported by National Science Foundation grant DMB 8816727, National Institute of General Medical Sciences grant GM 28994, and a predoctoral fellowship from the Department of Health and Human Services training grant GM 08352 to R.V.S.

\section{REFERENCES}

1. Allen, M. M. 1968. Simple conditions for growth of unicellular blue-green algae on plates. J. Phycol. 4:1-4.

2. Bancroft, I., C. P. Wolk, and E. V. Oren. 1989. Physical and genetic maps of the genome of the heterocyst-forming cyanobacterium Anabaena sp. strain PCC 7120. J. Bacteriol. 171: 5940-5948.

3. Belknap, W. R., and R. Haselkorn. 1987. Cloning and light regulation of expression of the phycocyanin operon of the cyanobacterium Anabaena. EMBO J. 6:871-884.

4. Bryant, D. A. 1982. Phycoerythrocyanin and phycoerythrin: properties and occurrence in cyanobacteria. J. Gen. Microbiol. 128:835-844.

5. Bryant, D. A. 1989. Genetic analysis of phycobilisome biosynthesis, assembly, structure, and function in the cyanobacterium Synechococcus sp. PCC 7002, p. 62-90. In S. E. Stevens and D. A. Bryant (ed.), Light energy transduction in photosynthesis: higher plant and bacterial models. American Society of Plant Physiologists, Rockville, Md.

6. Bryant, D. A., A. N. Glazer, and F. A. Eiserling. 1976. Characterization and structural properties of the major phycobiliproteins of Anabaena sp. Arch. Microbiol. 110:61-75.

7. Bryant, D. A., C. S. Hixson, and A. N. Glazer. 1978. Structural studies on phycobiliproteins. III. Comparison of bilin-containing peptides from the $\beta$ subunits of C-phycocyanin, R-phycocy- 
anin, and phycoerythrocyanin. J. Biol. Chem. 253:220-225.

8. Bryant, D. A., V. L. Stirewalt, M. Glauser, G. Frank, W. Sidler, and H. Zuber. 1991. A small multigene family encodes the rod-core linker polypeptides of Anabaena sp. PCC 7120 phycobilisomes. Gene 107:91-99.

9. Conley, P. B., P. G. Lemaux, T. L. Lomax, and A. R. Grossman. 1986. Genes encoding the major light-harvesting polypeptides are clustered on the genome of the cyanobacterium Fremyella diplosiphon. Proc. Natl. Acad. Sci. USA 83:3924-3928.

10. Costanzi, C., and D. Gillespie. 1987. Fast blots: immobilization of DNA and RNA from cells. Methods Enzymol. 152:582-587.

11. de Lorimier, R., R. L. Smith, and S. E. Stevens, Jr. Regulation of phycobilisome structure and gene expression by light intensity. Plant Physiol., in press.

12. de Lorimier, R., and S. M. Wilbanks. Unpublished data.

13. Duerring, M., R. Huber, W. Bode, R. Ruembeli, and H. Zuber. 1990. Refined three-dimensional structure of phycoerythrocyanin from the cyanobacterium Mastigocladus laminosus. J. Mol. Biol. 211:633-644.

14. Eberlein, M., and W. Kufer. 1990. Genes encoding both subunits of phycoerythrocyanin, a light harvesting biliprotein from the cyanobacterium Mastigocladus laminosus. Gene 94:133-136.

15. Füglistaller, P., F. Suter, and H. Zuber. 1983. The complete amino-acid sequence of both subunits of phycoerythrocyanin from the thermophilic cyanobacterium Mastigocladus laminosus. Hoppe-Seyler's Z. Physiol. Chem. 364:691-712.

16. Füglistaller, P., F. Suter, and H. Zuber. 1985. Linker polypeptides of the phycobilisome from the cyanobacterium Mastigocladus laminosus: amino-acid sequences and relationships. Biol. Chem. Hoppe-Seyler 366:993-1001.

17. Füglistaller, P., F. Suter, and H. Zuber. 1986. Linker polypeptides of the phycobilisome from the cyanobacterium Mastigocladus laminosus. I. Isolation and characterization of phycobiliprotein-linker-polypeptide complexes. Biol. Chem. Hoppe-Seyler 367:601-614.

18. Golden, S., J. Busslan, and R. Haselkorn. 1987. Genetic engineering of the cyanobacterial chromosome. Methods Enzymol. 153:215-231.
19. Lind, L. K., S. R. Kalla, A. Lönneborg, G. Öquist, and P. Gustafsson. 1987. Organization of the phycocyanin gene clusters in Anacystis nidulans. Acta Chem. Scand. Ser. B Org. Chem. Biochem. 41:112-115.

20. Mazel, D., J. Houmard, A. M. Castets, and N. Tandeau de Marsac. 1990. Highly repetitive DNA sequences in cyanobacterial genomes. J. Bacteriol. 172:2755-2761.

21. Mazel, D., J. Houmard, and N. Tandeau de Marsac. 1988. A multigene family in Calothrix sp. PCC7601 encodes phycocyanin, the major component of the cyanobacterial light harvesting antenna. Mol. Gen. Genet. 211:296-304.

22. Mazel, D., and P. Marliére. 1989. Adaptive eradication of methionine and cysteine from cyanobacterial light-harvesting proteins. Nature (London) 341:245-248.

23. Ong, L. J., and A. N. Glazer. 1987. R-Phycocyanin II, a new phycocyanin occuring in marine cyanobacterial species. Identification of the terminal acceptor bilin in phycocyanins. J. Biol. Chem. 262:6323-6327.

24. Sanger, F., S. Nicklen, and A. R. Coulson. 1977. DNA sequencing with chain-terminating inhibitors. Proc. Natl. Acad. Sci. USA 74:5463-5467.

25. Swanson, R. V. 1991. Ph.D. thesis. University of California, Berkeley, Calif.

26. Swanson, R. V., and A. N. Glazer. 1990. Separation of phycobiliprotein subunits by reverse-phase high-pressure liquid chromatography. Anal. Biochem. 188:295-299.

27. Swanson, R. V., L. J. Ong, S. M. Wilbanks, and A. N. Glazer. 1991. Phycoerythrins of marine unicellular cyanobacteria. II. Characterization of phycobiliproteins with unusually high phycourobilin content. J. Biol. Chem. 266:9528-9534.

28. Tandeau de Marsac, N., D. Mazel, V. Capuano, T. Damerval, and J. Houmard. 1990. Genetic analysis of the cyanobacterial light-harvesting antenna complex, p. 143-153. In G. Drews and E. A. Dawes (ed.), Molecular biology of membrane-bound complexes in phototrophic bacteria. Plenum Press, New York.

29. Wallace, R. B., and C. G. Miyada. 1987. Oligonucleotide probes for the screening of recombinant DNA libraries. Methods Enzymol. 152:432-442. 